\title{
Adaptive Request Scheduling for Parallel Scientific Web Services
}

\section{Heshan Lin}

Xiaosong Ma

Jiangtian $\mathrm{Li}$

Ting $Y_{u}$

Nagiza Samatova

North Carolina State Universty

Oak Ridge National Laboratory

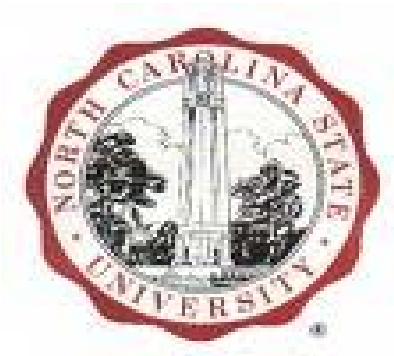




\section{"Data Rich" Scientific Applications}

- Scientists need to routinely process hundreds of GBS or TBS of data

- Biology, cosmology, climate

- Public science data grow rapidly

- E.g., GenBank size grows > 5 orders of magnitude in last 2 decades

- Storing, analyzing such data beyond capacity of personal computers

Growth of GenBank

(1982 - 2005)

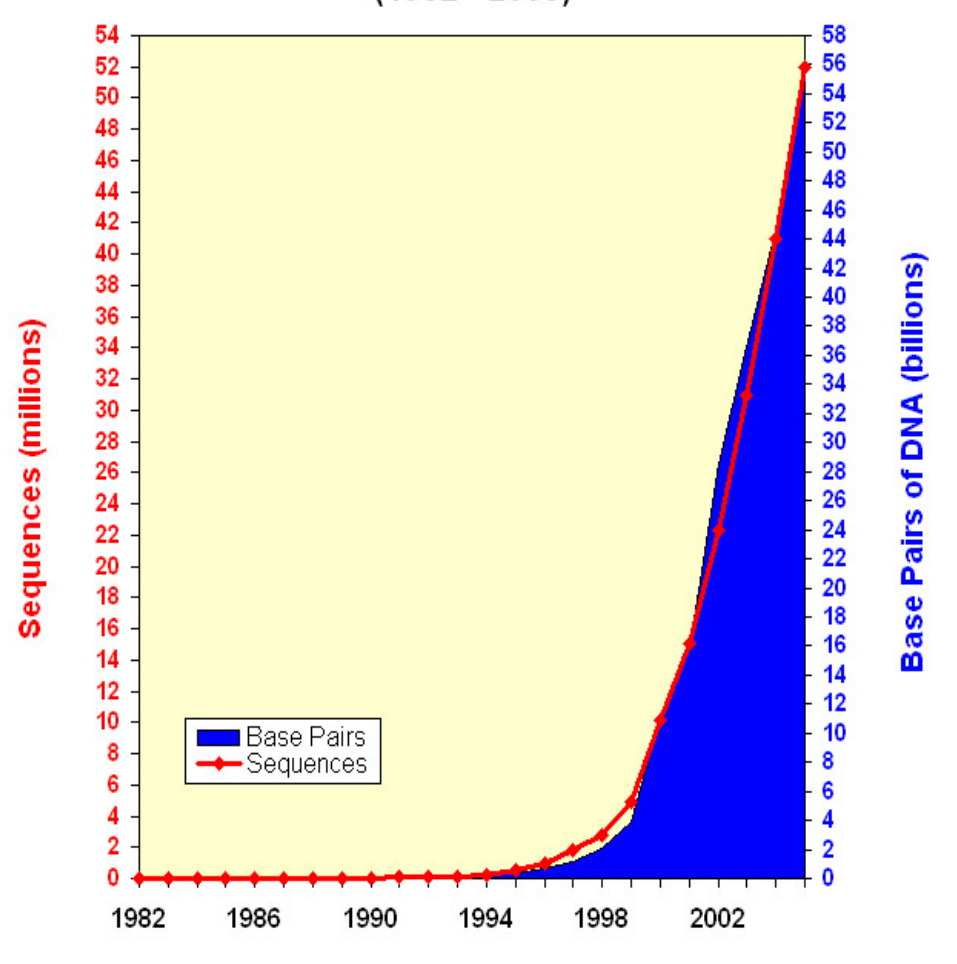




\section{Scientific Web Services}

- Increasingly popular to address data growth

- Efficient sharing of

- public data repository

- high-end computing resources

- Hiding parallel job management overhead
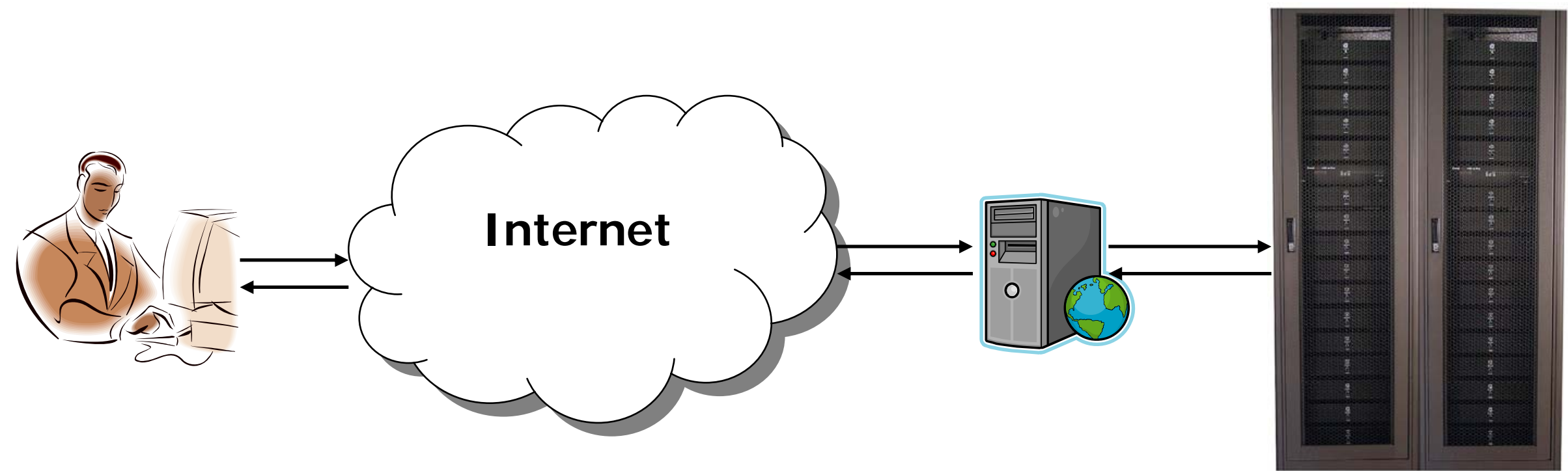


\section{New Scheduling Context}

- Characteristics of scientific requests

- Compute-intensive: require processing on multiple processors

- Data-intensive: accessing GBs to TBs of data

- Related scheduling studies

- Content serving cluster web server: focusing on data-locality

- Space sharing parallel job scheduling: focusing on parallel efficiency

- Needs computation- and data-aware scheduling algorithms 


\section{Our Contributions}

- Two-level adaptive scheduling framework for scientific web services

- Goal: to improve average request response time

- Takes into account both data-locality and parallel efficiency

- Automatically adapts to system loads and request patterns

- Case study: genomic sequence similarity search (BLAST) web server

- Performance evaluation on real cluster 


\section{Road Map}

- Introduction

- Background

- Scheduling design

- Experiment results

- Conclusions 


\section{BLAST}

- Routinely used in many biomedical researches

- Search similarities between query sequences and those in sequence database

- Predict structures and functions of new sequences

- Verify experiment and computation results

- Analogous to web search engines (e.g. Google)

\begin{tabular}{|l|l|l|}
\hline & \multicolumn{1}{|c|}{ Web Search Engine } & \multicolumn{1}{c|}{ BLAST } \\
\hline Input & Key word(s) & Query sequence(s) \\
\hline Search space & Internet & Known sequence database \\
\hline Output & Related web pages & $\begin{array}{l}\text { DB sequences similar to } \\
\text { the query }\end{array}$ \\
\hline Sorted by & Closeness \& rank & Score (Similarity) \\
\hline
\end{tabular}




\section{Road Map}

- Introduction

- Background

- Scheduling algorithm design

- Experiment results

- Conclusions 


\section{System Architecture}

- Front end node

- Receives request and making scheduling decision

- Backend nodes

- Perform parallel BLAST jobs

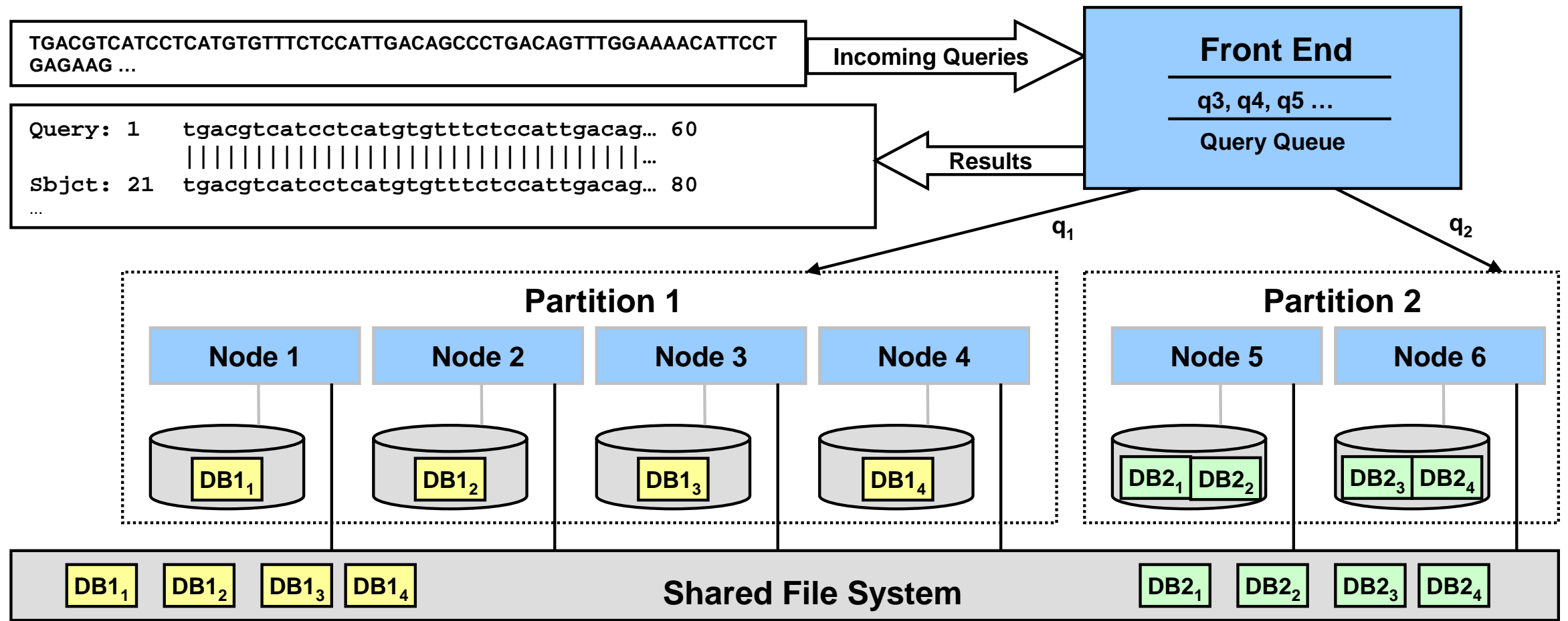




\section{Overview}

- Scheduling problem: find partition of cluster to service request

- How many processors to allocate?

- And on which processors?

- Which database fragment(s) to search on each processor?

- Scheduling techniques

- Efficiency-oriented scheduling

- Data-oriented scheduling

- Challenge: to automatically adapt to system loads and query patterns 


\section{Efficiency-Oriented Scheduling}

- Response time = wait time + service time

- Intuition

- Partition size grows $\Rightarrow$ speedup increases, efficiency decreases

- When load light, use large partition size $\rightarrow$ reduce service time

- When load heavy, use small partition size $\rightarrow$ reduce wait time

- MAP [Dandamudi99]

- Compute partition size

- S: number of jobs being serviced

- $f$ : adjustable parameter $(0<=f<1)$

$$
\text { partition_size }=\operatorname{Max}\left(1, \text { ceil }\left(\frac{\text { total_processors }}{\text { queue_length }+1+f * S}\right)\right)
$$




\section{Our Solution: RMAP}

- Define a range of partition sizes $\left[P_{\min }, P_{\max }\right]$ for each $D B$

- $P_{\min }$ : smallest \# procs whose aggregate memory can hold the database

- $P_{\max }$ : saturation point of speedup curve
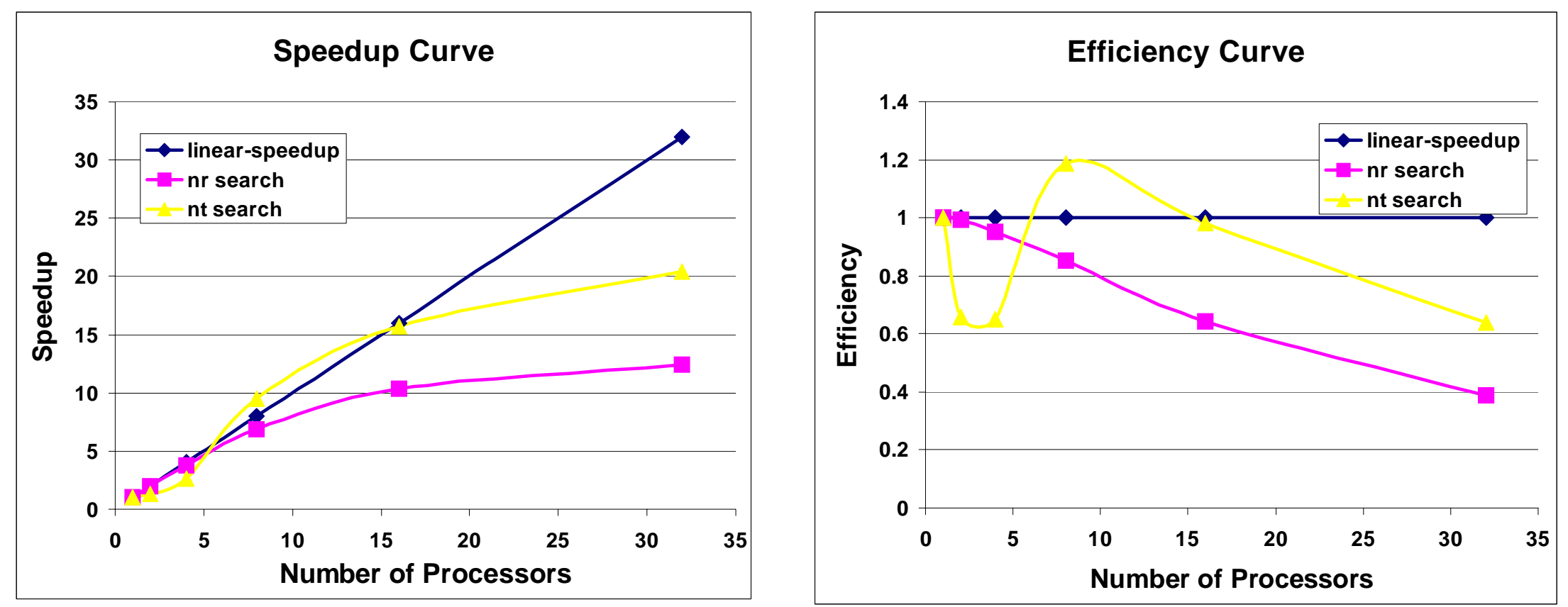


\section{Data-Oriented Scheduling}

- Given partition size p, which processors should search next query?

- Naïve approach

- FA (First Available): similar to batch job scheduling

- Orders processors by rank, pick first $p$ idle processors

- Does not consider data locality

- LARD algorithm for cluster web servers [Pai98]

- Intuition: assigns object request to processor that recently serviced it

- Considers both data locality and load balance 


\section{Data-Oriented Scheduling (cont.)}

- What's new here?

- Servicing a query requires co-scheduling of multiple nodes

- A processor can only serve one query at a time

- Our solution: PLARD

- Multiple queues and processor pools

- Per-database basis

- Query assignment and load balancing among processor pools

- Assign and migrate processors in groups 


\section{PLARD + RMAP}

- Two-level scheduling decisions

- Inter-DBPool: dynamically adjusting DB pool sizes guided by RMAP on global statss

- Inner-DBPool: RMAP on local (per DB) stats

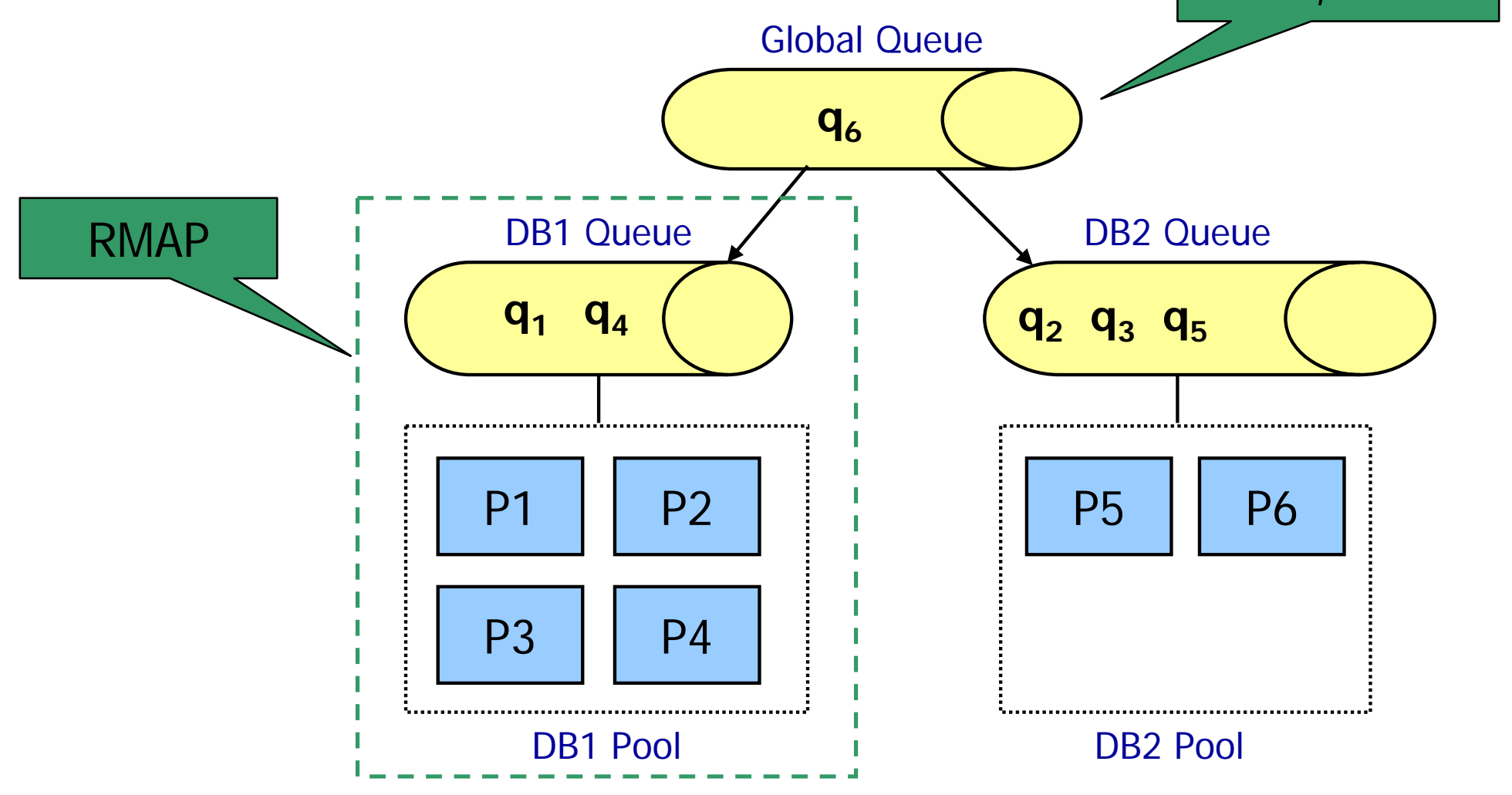




\section{Road Map}

- Introduction

- Background

- Scheduling design

- Experiment results

- Conclusions 


\section{Experiment Setup}

- Input data

- 5 NCBI sequence databases

- Synthesized query trace, Poisson arrivals

- 1000 randomly sampled query sequences (proportional to DB size)

- Backend cluster

- 32 Xeon procs, Linux OS, Gigabit Ethernet

\begin{tabular}{|c|c|c|c|c|c|}
\hline DB Name & Type & Raw Size & $\begin{array}{c}\text { Formatted } \\
\text { Size }\end{array}$ & $\boldsymbol{P}_{\min }$ & $\boldsymbol{P}_{\max }$ \\
\hline env_nr & $\mathrm{P}$ & $1.7 G \mathrm{~B}$ & $2.5 G \mathrm{~B}$ & 2 & 32 \\
\hline nr & $\mathrm{P}$ & $2.6 \mathrm{~GB}$ & $3.0 G \mathrm{~B}$ & 4 & 32 \\
\hline est_mouse & $\mathrm{N}$ & $2.8 \mathrm{~GB}$ & $2.0 G \mathrm{~B}$ & 2 & 16 \\
\hline nt & $\mathrm{N}$ & $21 G \mathrm{~B}$ & $6.5 G \mathrm{~B}$ & 8 & 32 \\
\hline gss & $\mathrm{N}$ & $16 \mathrm{~GB}$ & $9.16 \mathrm{~B}$ & 8 & 32 \\
\hline
\end{tabular}




\section{PLARD Impacts}

\section{Avg \#Page Faults}
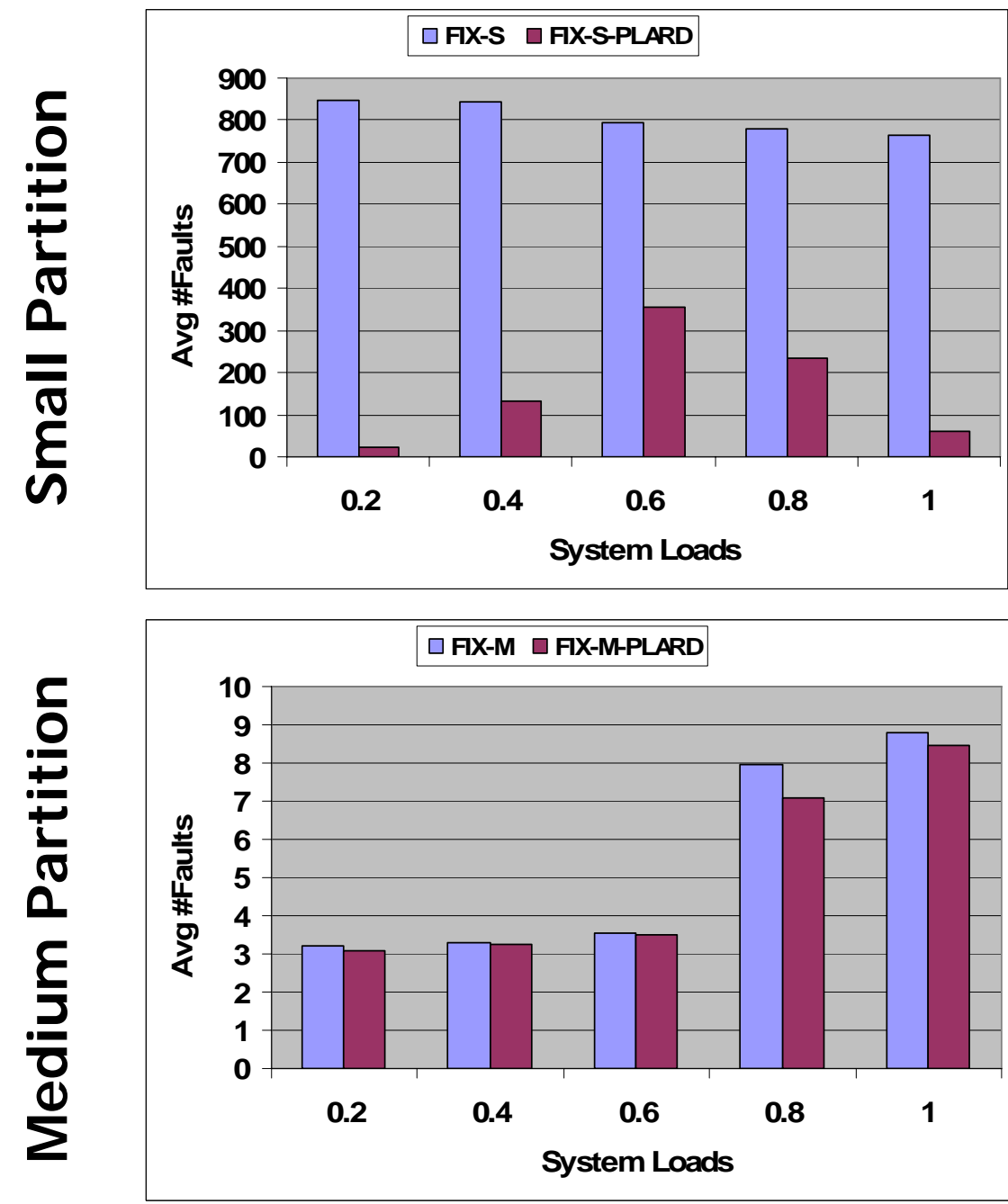

\section{Avg Response Time (Log)}
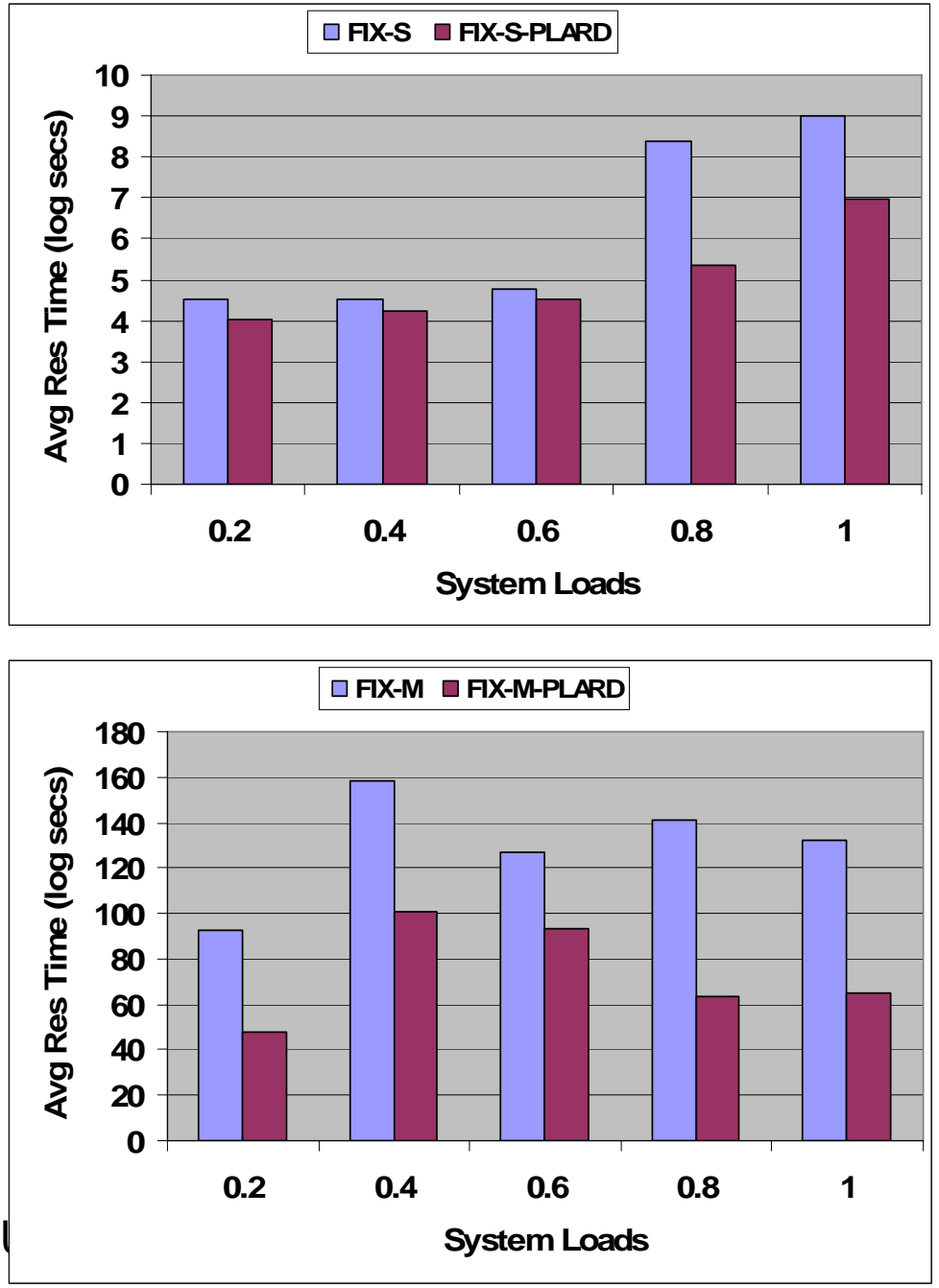


\section{PLARD Impacts (cont.)}

- Count \# of searched queries on each processor

- PLARD results in more balanced loads across processors
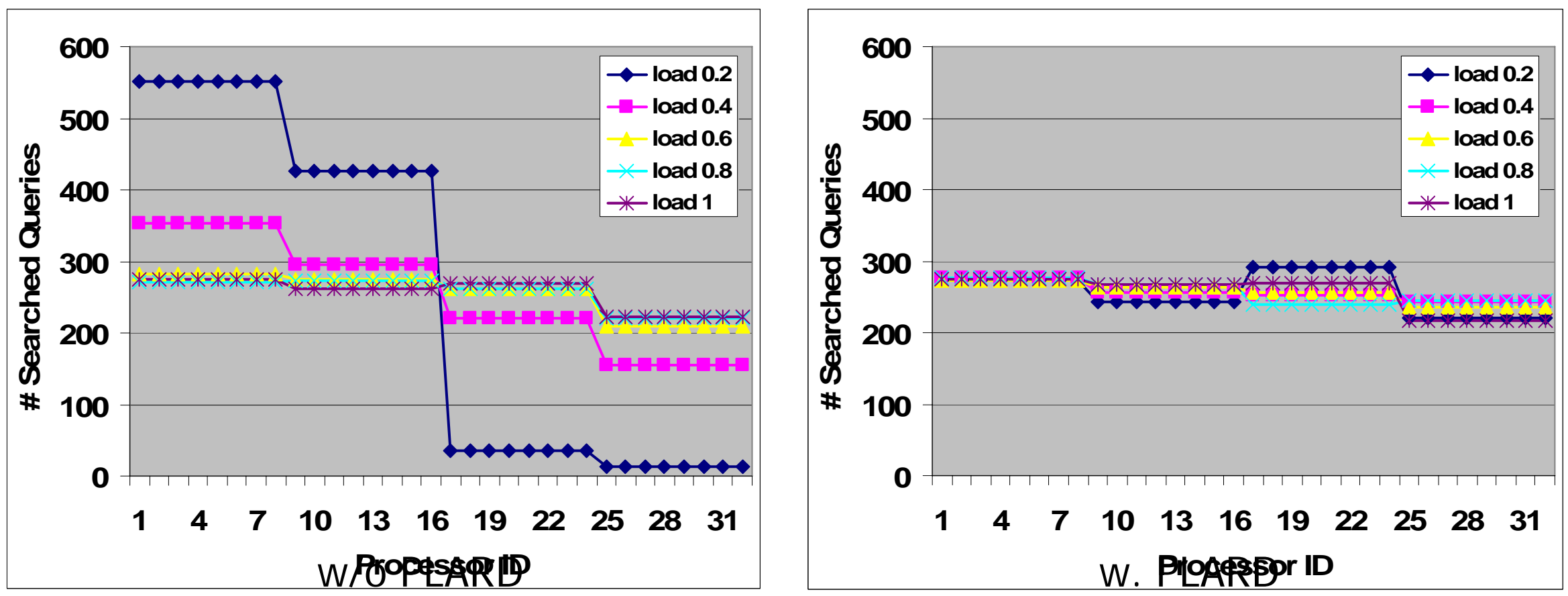


\section{Adaptive to Fixed Arrival Rates}

- Static policies work well for certain workload

- RMAP wins across the board

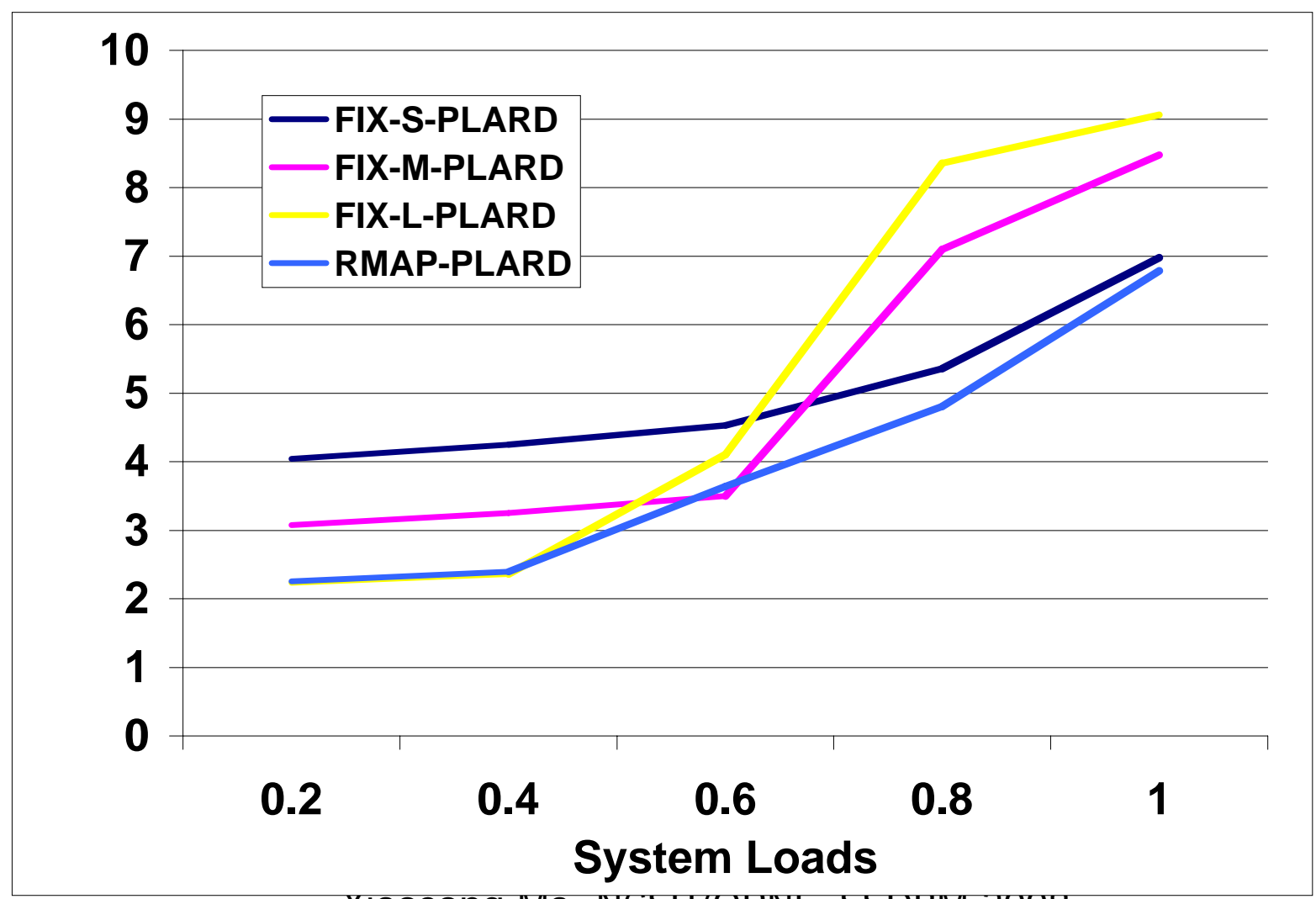




\section{Adaptive to Mixed Arrival Rates}

- Two traces with mixed arrival rates

- Trace 1: $0.2+0.4+0.6+0.8$

- Trace 2: $0.2+0.8+0.4+1.0$

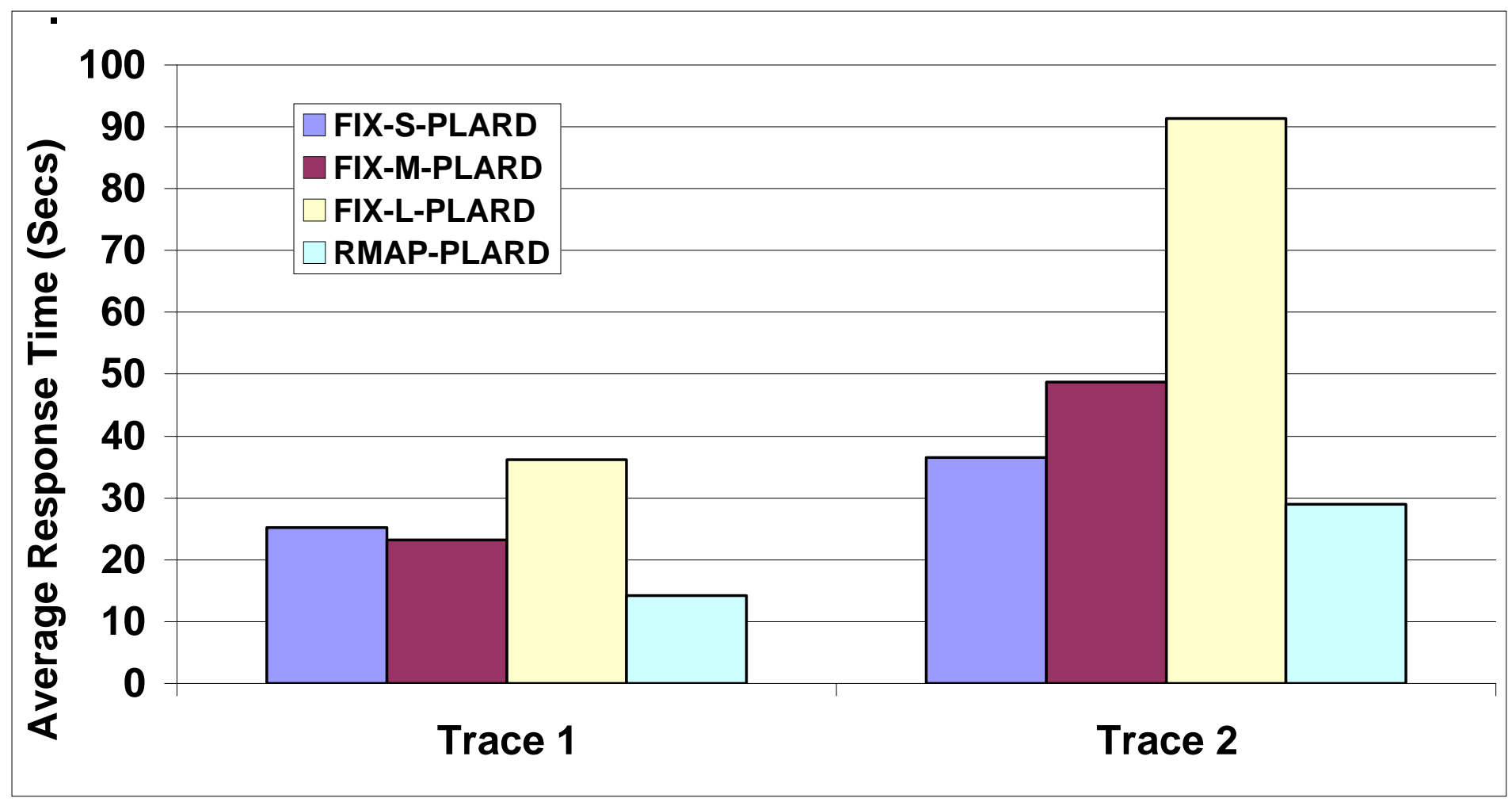




\section{Road Map}

- Introduction

- Background

- Scheduling design

- Experiment results

- Conclusions 


\section{Conclusions}

- Scientific web service request scheduling not well studied

- "Moldable jobs" realized

- Two-level adaptive scheduling framework

- RMAP: parallel efficiency aware

- PLARD: data locality aware

- Combined adaptive policy autonomically adapts to system loads and query patterns 


\section{References}

- [Dandamudi99] S. Dandamudi and H. Yu. Performance of adaptive space sharing processor allocation policies for distributed-memory multicomputers. JPDC, 58(1), 1999.

- [Gardner06], M. Gardner, W. Feng, J. Archuleta, H. Lin, and X. Ma, Parallel Genomic Sequence-Searching on an Ad-Hoc Grid: Experiences, Lessons Learned, and Implications. Supercomputing, 2006

- [Lin05], H. Lin, X. Ma, P. Chandramohan, A. Geist, and N. Samatova, Efficient Data Access for Parallel BLAST, IPDPS, 2005.

- [Lin08], H. Lin, P. Balaji, R. Pool, C. Sosa, X. Ma, and W. Feng, Massively Parallet'Genomic Sequence Search on the BlueGene/P Architecture. To appear, Supercomputing, 2008.

- [Pai98] V. Pai, M. Aron, G. Banga, M. Svendsen, P. Druschel, $W$. Zwaenepoel, and $E$. Nahum. Locality-aware request distribution in cluster-based network servers. ASPLOS-VIII, 1998. 


\section{Thank You}

- Questions? 